\title{
Modular Interacting Multiple Model Based on Extended Viterbi Algorithm for Maneuvering Target Tracking
}

\author{
Xia Liu, Fei Long, Wenjie Zhang, and Lu Guo \\ College of Big Data and Information Engineering, Guizhou University, Guiyang 550025, China \\ Correspondence should be addressed to Fei Long; flong1973@aliyun.com
}

Received 25 September 2014; Accepted 10 January 2015

Academic Editor: Jian-Ming Lu

Copyright (C) 2015 Xia Liu et al. This is an open access article distributed under the Creative Commons Attribution License, which permits unrestricted use, distribution, and reproduction in any medium, provided the original work is properly cited.

\begin{abstract}
A new maneuvering target tracking algorithm is investigated, which is modeled as a class of Markov jump linear systems (MJLS). Drawing on the experience of combination idea of the extended Viterbi algorithm (EV) and the interacting multiple model algorithm (IMM), a modular interacting multiple model based on extended Viterbi (MIMMEV) is presented. The MIMMEV algorithm consists of $N$ independent interacting multiple model-extended Viterbi (IMM-EV). Furthermore, these IMM-EV filters are independent and working in parallel in the MIMMEV algorithm. According to the derived probability, the estimated state of every moment is the weighted sum of each estimator at the corresponding time. Simulation results demonstrate that the proposed algorithm improves the tracking precision and reduces the computational burden compared with traditional IMM and IMM-EV.
\end{abstract}

\section{Introduction}

Many researches have been undertaken in the field of estimation theory over the past decades. In both military and civilian fields, such as air defense and air-traffic control, obtaining a reliable and accurate mode in targets tracking has been the main objective in the design of target tracking system [1].

According to the number of target motion model, the maneuvering target tracking algorithm can be divided into single model algorithm and multiple model (MM) algorithm. Considering the characteristics of maneuvering target movement, using a single and fixed model has a difficulty in describing the motion state of target accurately. As a result, MM algorithm is formed, which uses multiple models to describe the movement of maneuvering targets at the same time. Because of powerful function and robustness, MM algorithm has become an important approach in the target tracking estimate. It has a better effect in tracking nonmaneuvering targets but worse for maneuvering target tracking. Early MM algorithm belongs to static interactive multiple model algorithm without input, due to inconsideration of model switching. Blom and Barshalom had put forward a traditional IMM with Markov switching coefficients. IMM does not need to test maneuvering but needs to adjust the probability of each model to implement switching among models in order to achieve the overall adaptation. In fact, the generalized pseudo-Bayesian (GPB) algorithm and the interacting multiple model (IMM) algorithm are popular methods. Compared with GPB, IMM has a better performance in the calculation; thus, IMM has been used widely [2].

The IMM algorithm, which employs a fixed set of models, always works well for problems with a small model set. Nevertheless, many practical problems involve much more models [3]. The effect of using too many models is as bad as the case of too few models [4]. Actually, the performance will decline if too many models are used due to redundant "competition" from the "unnecessary" models.

Some modified IMM algorithms were presented for improving performance or computation efficiency in recent years [5-7]. However, a prodigious defect is the fixed model set that it has. A variable structure MM (namely, VSMM) algorithm is proposed to solve the dilemma where the model set not only differs across targets but also varies with time for given targets $[8,9]$. Not only does VSMM inherit the effective cooperation strategies of the IMM, but also it adapts to the outworld by generating new filters if the existing ones are not good and by removing those filters which are harmful. The estimation with VSMM, however, depends on the auxiliary 
information (such as the terrain topography). Thus, it is very hard to realize the VSMM without the auxiliary information. The mechanism of the extended Viterbi (EV) algorithm is similar to those of VSMM, and it finds $m(1 \leq m \leq n$, and $n$ is the number of all paths) potentially most likely paths. Above all, the estimation of EV does not depend on the auxiliary information. As a result, an extended Viterbi based interacting multiple model algorithm, namely, IMM-EV, is presented as the incorporation of some functional mechanisms of EV and IMM. IMM-EV with some appropriate choice of $m$ can improve IMM and VSMM in terms of its performance and computation [10]. In fact, there is a case that some IMMEV may produce more accurate state estimation during some maneuvering target tracking while work worse during others. On these conditions, a MIMMEV algorithm is presented in this paper. The proposed algorithm consists of $N$ IMM-EV filters which are mutually independent. The state estimation equals mixing of all module estimations according to the module probability.

The remainder of the paper is organized as follows. Section 2 briefly reviews the IMM algorithm and EV algorithm. In Section 3, the MIMMEV algorithm is proposed. The MIMMEV algorithm contains $N$ independent IMM-EV filters performing in parallel, and it is suitable for the large model set. A simulation for testing the proposed algorithm is performed in Section 4. Finally, some conclusions are given in Section 5.

\section{Preliminaries}

2.1. The IMM Algorithm. Because of its powerful function and robustness, $\mathrm{MM}$ estimation is a powerful approach to target tracking. The IMM algorithm, as one of MM algorithms, has been widely used in target tracking. The IMM includes $n$ interacting filters working in parallel and each filter is marked as $M^{(j)}, j=1,2, \ldots, n$. Assume that the movement characteristics of the target at time $k$ is expressed as $M(k)$, and $n$ models in IMM algorithm can describe any possible target motion characteristics; namely, $M(k) \in\left\{M^{(j)}\right\}_{j=1}^{n}$. Model conversion is assumed to be a Markov process with known transition probability; namely,

$$
p_{i j}=p\left\{M(k)=M^{(j)} \mid M(k-1)=M^{(i)}\right\},
$$

where $p_{i j}$ is the transition probability from model $i$ to model $j$ according to the Markov chain. Based on above, the posterior probability is calculated when each model is valid. Then the final target estimation can be represented with the weighted sum of each model which is effective, and the weighted factors are posterior probabilities computed above. IMM algorithm includes five steps [11]. It can be known that the performance of IMM algorithm is much better than one of single model algorithm [8]. However, the performance of IMM will deteriorate if too many models are utilized owing to the excessive competition from the redundant models. Two models is the best solution in the normal cases when model transition matrix is effective.

2.2. The EV Algorithm. In order to solve the above dilemma that the IMM algorithm faces, those unnecessary filtering models in the model set need be removed; thus, the VSMM algorithm arises at the historic moment. The ideal of the VSMM is to select the effective models in the model set to realize optimization of the algorithm. Nevertheless, VSMM has a drawback that smooth realization of VSMM depends on auxiliary information. The good news is that the mechanism of the extended Viterbi (EV) algorithm is similar to those of VSMM. The EV algorithm can find a solution to the above problem. The mechanism of the EV algorithm is to search for $m$ most likely model paths out of $n$ given models at each instant. A more detailed exposition about the EV algorithm is provided in $[12,13]$. Some functional mechanisms of the EV algorithm can be incorporated into the IMM algorithm for hypothesis reductions, so IMM-EV is presented.

\section{The Modular Interacting Multiple Model Based on Extended Viterbi Algorithm}

3.1. The IMM-EV Algorithm. On the one hand, like the IMM algorithm, an IMM-EV algorithm does spread estimates produced from all models at all times. On the other hand, like the VSMM algorithm, an IMM-EV algorithm may mix only those estimates of effective models out of all models at given times to get the state estimate of a system and then potentially avoid the loss of accuracy because of overmodeling. IMM-EV algorithm includes $N$ interacting filters operating in parallel; one cycle of the algorithm is briefly described as follows.

In this subsection, the following notation will be used: give $u_{0}(j)=\eta_{j}, 0 \leq \eta_{j} \leq 1$, and $\sum_{j=1}^{n} \eta_{j}=1$ and give $\widehat{x}_{0}^{j}, P_{0}^{j}$, where $\eta_{j}$ is the probability of mode $j(j=1 \cdots n)$ [14].

Step 1. Calculate mixing probabilities

$$
\begin{gathered}
u_{k-1}\left(l_{s j} \mid j\right)=\frac{\max _{1 \leq i \leq n}{ }^{(s)}\left\{p_{i j} u_{k-1}(i)\right\}}{\sum_{s=1}^{m} \max _{1 \leq i \leq n}{ }^{(s)}\left\{p_{i j} u_{k-1}(i)\right\}}, \\
l_{s j}=\arg \left\{\max _{1 \leq i \leq n}^{(s)}\left\{p_{i j} u_{k-1}(i)\right\}\right\},
\end{gathered}
$$

where $u_{k-1}(\cdot \mid j)$ is a mixing probability at the time instant $k-1$.

Step 2. Calculate mixed state estimates $\widehat{X}_{k-1}^{0 j}$ and state error covariance matrices $P_{k-1}^{0 j}$ as follows:

$$
\begin{aligned}
\widehat{X}_{k-1 \mid k-1}^{0 j}= & \sum_{s=1}^{m} \widehat{X}_{k-1 \mid k-1}^{l_{s j}} u_{k-1}\left(l_{s j} \mid j\right) \\
P_{k-1 \mid k-1}^{0 j}=\sum_{s=1}^{m} u_{k-1}\left(l_{s j} \mid j\right) & \\
\cdot & \left\{P_{k-1 \mid k-1}^{l_{s j}}+\left[\widehat{X}_{k-1 \mid k-1}^{l_{s j}}-\widehat{X}_{k-1 \mid k-1}^{0 j}\right]\right. \\
& \left.*\left[\widehat{X}_{k-1 \mid k-1}^{l_{s j}}-\widehat{X}_{k-1 \mid k-1}^{0 j}\right]^{\prime}\right\} .
\end{aligned}
$$




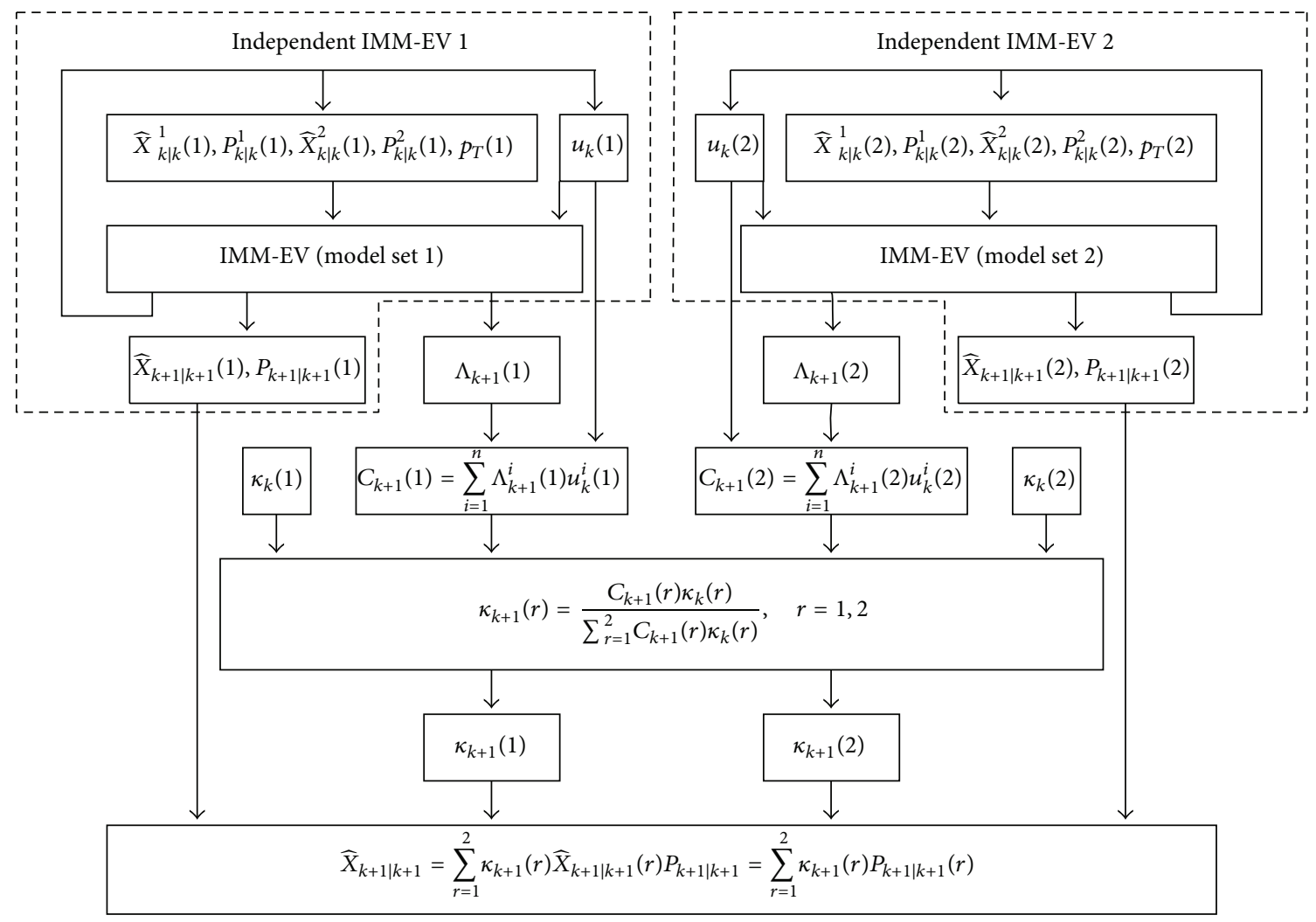

Figure 1: MIMMEV algorithm $(n=2, N=2)$.

Step 3. It is model filtering. This step is the same as IMM.

Step 4. Update model probabilities as follows:

$$
u_{k}(j)=\frac{\Lambda_{k}(j) \sum_{s=1}^{m} \max _{1 \leq i \leq n}(s)\left\{p_{i j} u_{k-1}(i)\right\}}{\sum_{j=1}^{n} \Lambda_{k}(j) \sum_{s=1}^{m} \max _{1 \leq i \leq n}(s)\left\{p_{i j} u_{k-1}(i)\right\}},
$$

where $\Lambda_{k}^{j}$ is the likelihood function of the model $j$ at the time instant $k$, and $\Lambda_{k}^{j}=N\left[v_{k}^{j} ; 0 ; S_{k}^{j}\right]$.

Step 5. Calculate $m$ largest model probabilities as follows:

$$
\begin{aligned}
\tilde{u}_{k}\left(\tilde{l}_{r}\right) & =\frac{\max _{1 \leq j \leq n}{ }^{(r)}\left\{u_{k}(j)\right\}}{\sum_{r=1}^{m} \max _{1 \leq j \leq n}{ }^{(r)}\left\{u_{k}(j)\right\}}, \\
\tilde{l}_{r} & =\arg \left\{\max _{1 \leq j \leq n}^{(r)}\left\{u_{k}(j)\right\}\right\} .
\end{aligned}
$$

Step 6. Output of state estimate is as follows:

$$
\widehat{x}_{k}=\sum_{r=1}^{m} \widetilde{u}_{k}\left(\widetilde{l}_{r}\right) \tilde{x}_{k}^{\tilde{l}_{r}}
$$

When $m=n$, the IMM-EV algorithm is equivalent to the IMM algorithm. The IMM-EV algorithm is derived from the IMM algorithm and thus they have some similar functional parts. Their difference is that the development of an IMM-EV algorithm is on the basis of the EV algorithm.

3.2. The MIMMEV Algorithm. There is such a situation that some IMM-EV algorithms may work well during constantvelocity tracking while other IMM-EV algorithms may perform well during maneuver tracking. Aiming at the above situation, the MIMMEV algorithm is put forward in this paper. The MIMMEV algorithm contains $N$ independent IMM-EV filters performing in parallel, as $N$ independent modules. Independence between different IMM-EV filters can remove the excessive competition from the unnecessary models. The estimated state of every moment is the weighted sum of each module at the corresponding time according to the derived probability of each IMM-EV filter. Figure 1 shows the flow chart for one cycle of the MBIMMEV logic. The MBIMMEV algorithm includes four fundamental steps.

Step 1. There is the parallel independent IMM-EV:

$\widehat{X}_{k+1 \mid k+1}(r), P_{k+1 \mid k+1}(r), u_{k+1}(r)$ and $\Lambda_{k+1}(r)$ are computed for each model set using IMM-EV. 
Step 2. The likelihood function value of every model set is as follows:

$$
C_{k+1}(r)=\sum_{j=1}^{n} \Lambda_{k+1}^{j}(r) u_{k}^{j}(r), \quad r=1, \ldots, N
$$

Step 3. Normalized likelihood function value of every model set is as follows:

$$
\kappa_{k+1}(r)=\frac{C_{k+1}(r) \kappa_{k}(r)}{\sum_{r=1}^{N} C_{k+1}(r) \kappa_{k}(r)}, \quad r=1, \ldots, N
$$

In (8), $\kappa_{k}(r)$ is the weight probability of model set $r$ at the time instant $k$.

Step 4. Output of state estimate is as follows:

$$
\widehat{X}_{k+1 \mid k+1}=\sum_{r=1}^{N} \kappa_{k+1}(r) \widehat{X}_{k+1 \mid k+1}(r)
$$

From the above explanation, we guarantee the independence of all IMM-EV filters; thus, the proposed algorithm can solve the deterioration of performance due to the excessive competition from the unnecessary models.

\section{Simulation}

The given algorithm is illustrated at length with one example of complex maneuvering target tracking, and the sampling period $T$ is $1 \mathrm{~s}$. The trajectory is a target moving in the $(x, y)$ plane with an initial position $[10 \mathrm{~km}, 40 \mathrm{~km}]^{\prime}$ and an initial velocity $[0 \mathrm{~m} / \mathrm{s},-300 \mathrm{~m} / \mathrm{s}]^{\prime}$. The trajectory is shown in Figure 2 that implements 9 motions, namely, CV, CA, CV, CA, $\mathrm{CV}, \mathrm{CT}, \mathrm{CV}, \mathrm{CT}$, and $\mathrm{CV}$, as follows:

(1) CV motion in $50 \mathrm{~s}$;

(2) CA motion in $15 \mathrm{~s}$ with an acceleration $\left(20 \mathrm{~m} / \mathrm{s}^{2}\right.$, $\left.20 \mathrm{~m} / \mathrm{s}^{2}\right)$;

(3) CV motion in $50 \mathrm{~s}$;

(4) CA motion in $10 \mathrm{~s}$ with an acceleration $\left(-30 \mathrm{~m} / \mathrm{s}^{2}\right.$, $\left.-30 \mathrm{~m} / \mathrm{s}^{2}\right)$;

(5) CV motion in $50 \mathrm{~s}$;

(6) coordinated turn motion in $16 \mathrm{~s}$ with a radius $1.5 \mathrm{~km}$ and an acceleration $60 \mathrm{~m} / \mathrm{s}^{2}$;

(7) CV motion in $50 \mathrm{~s}$;

(8) coordinated turn motion in $16 \mathrm{~s}$ with a radius $1.5 \mathrm{~km}$ and an acceleration $60 \mathrm{~m} / \mathrm{s}^{2}$ and

(9) CV motion in $50 \mathrm{~s}$.

The MIMMEV algorithm contains two modules (CVCA2 and CVCT2). The first module includes three models:

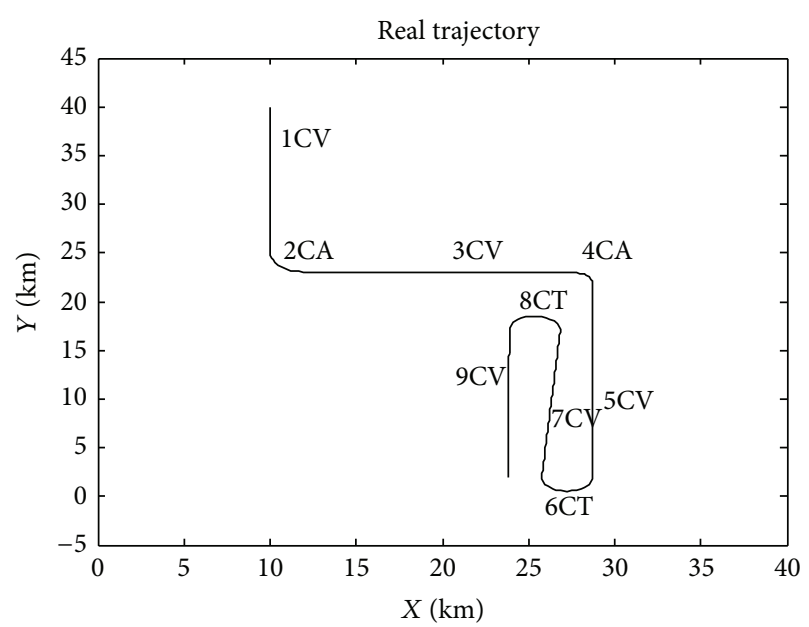

FIgURE 2: Target trajectory.

CV model, CA model with small process noise covariance, and CA model with large process noise covariance [15]. The second module includes three models: CV motion, clockwise CT, and counterclockwise CT model with known turn rates. The two modules are to search for $m=2$ most likely model paths out of $n=3$ given models at each instant. The standard covariance of models' process noise is $\sigma=2 \mathrm{~m} / \mathrm{s}$, the standard deviation of measurement noise is $\sigma x=\sigma y=50 \mathrm{~m} / \mathrm{s}$, and the matrix of the probabilities is

$$
P=\left[\begin{array}{ccc}
0.95 & 0.025 & 0.025 \\
0.025 & 0.95 & 0.025 \\
0.025 & 0.025 & 0.95
\end{array}\right]
$$

The RMSEs of IMM, IMM-EV, and MIMMEV are demonstrated in Figure 3. During CV and CA motion, the performances of the three algorithms are similar, but, during coordinated turn motion, MIMMEV algorithm has better performance than the other two. The RMSE of MIMMEV is the least on the whole. It proves that MIMMEV algorithm proposed has better performance than IMM and IMMEV. The proposed algorithm is a viable algorithm to other tracking methods.

\section{Conclusion}

The MIMMEV algorithm contains independent modules, which are independent IMM-EV filters performing in parallel. According to the derived probability, the estimated state of every moment equals the weighted sum of each estimator at the corresponding time. The presented algorithm inherits the merit of the IMM-EV methods. The MIMMEV algorithm improves performance of maneuvering target tracking by 


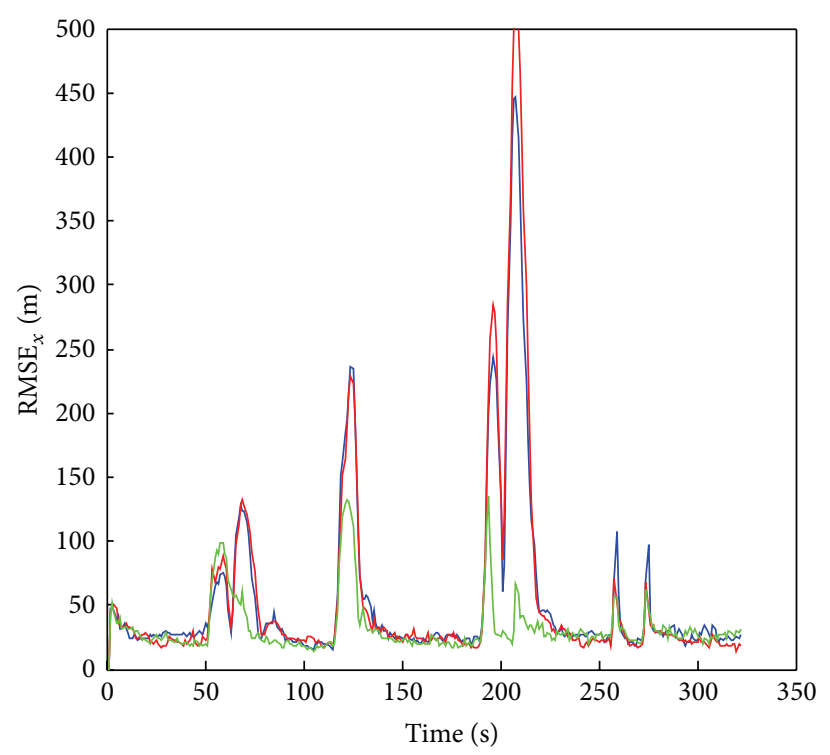

(a)

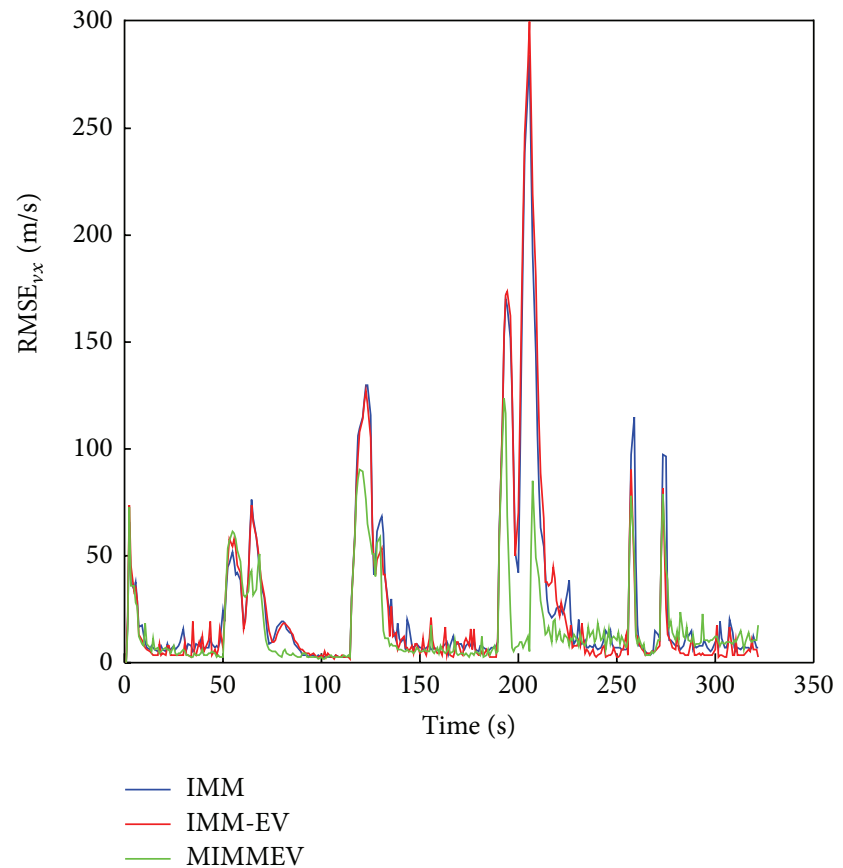

(c)

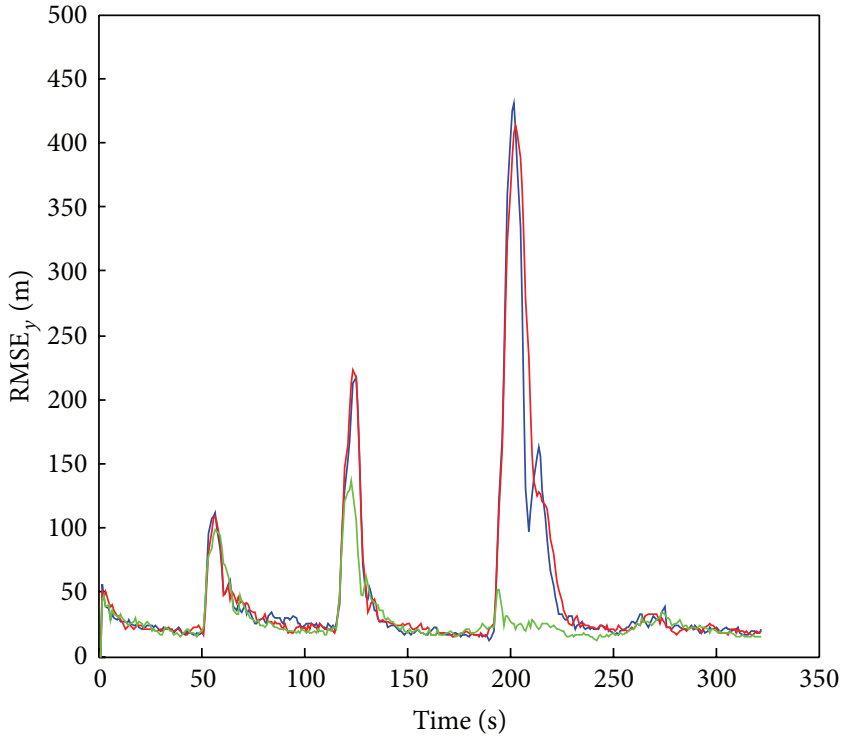

(b)

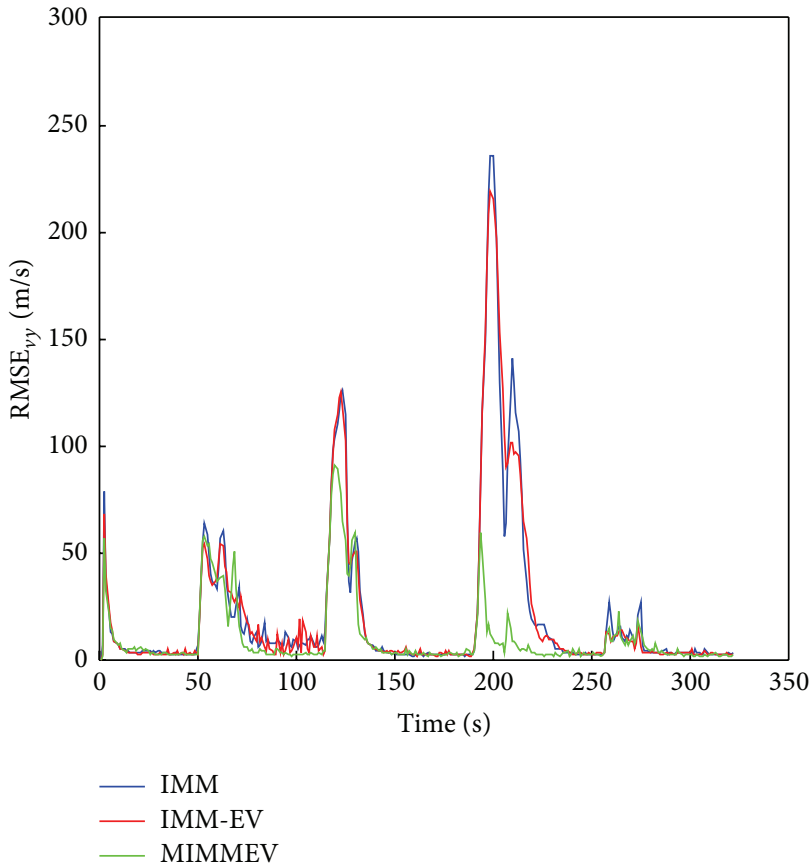

(d)

FIgure 3: Position RMSE and velocity RMSE for IMM, IMM-EV, and MIMME.

avoiding the excessive competition from the unnecessary models. And then it reduces computation burden effectively.

\section{Conflict of Interests}

The authors declare that there is no conflict of interests regarding the publication of this paper.

\section{Acknowledgments}

This work is supported by National Natural Science Foundation of China under Grant no. 61263005, the Research Fund for the Doctoral Program of Higher Education under Grant no. 20105201120003, and the Program for New Century Excellent Talents in Chinese University under Grant no. NCET-120657.

\section{References}

[1] E. Mazor, A. Averbuch, Y. Bar-Shalom, and J. Dayan, "Interacting multiple model methods in target tracking: a survey," IEEE Transactions on Aerospace and Electronic Systems, vol. 34, no. 1, pp. 103-123, 1998.

[2] H. Oh, H.-S. Shin, S. Kim, A. Tsourdos, and B. A. White, "Airborne behaviour monitoring using Gaussian processes with 
map information," IET Radar, Sonar and Navigation, vol. 7, no. 4, pp. 393-400, 2013.

[3] J. Ru and X. R. Li, "Variable-structure multiple-model approach to fault detection, identification, and estimation," IEEE Transactions on Control Systems Technology, vol. 16, no. 5, pp.1029-1038, 2008.

[4] L. Bloomer and J. E. Gray, "Are more models better? The effect of the model transition matrix on the IMM filter," in Proceedings of the 34th IEEE Southeastern Symposium on System Theory, pp. 20-25, IEEE, 2002.

[5] L. A. Johnston and V. Krishnamurthy, "An improvement to the interacting multiple model (IMM) algorithm," IEEE Transactions on Signal Processing, vol. 49, no. 12, pp. 2909-2923, 2001.

[6] N. Bergman, A. Doucet, and N. Gordon, "Optimal estimation and Cramer Rao bounds for partial non-Gaussian state space models," Annals of the Institute of Statistical Mathematics, vol. 53, no. 1, pp. 97-112, 2001.

[7] G. W. Pulford and B. F. La Scala, "MAP estimation of target manoeuvre sequence with the expectation-maximization algorithm," IEEE Transactions on Aerospace and Electronic Systems, vol. 38, no. 2, pp. 367-377, 2002.

[8] X. R. Li, "A survey of maneuvering target tracking-part II: model set adaptation," IEEE Transactions on Automatic Control, vol. 45, no. 11, pp. 2047-2060, 2000.

[9] X. R. Li, X. R. Zhi, and Y. Zhang, "A survey of maneuvering target tracking-part III: model-group switching algorithm," IEEE Transactions on Aerospace and Electronic Systems, vol. 35, no. 1, pp. 225-241, 1999.

[10] T.-J. Ho and B.-S. Chen, "Novel extended Viterbi-based multiple-model algorithms for state estimation of discrete-time systems with Markov jump parameters," IEEE Transactions on Signal Processing, vol. 54, no. 2, pp. 393-404, 2006.

[11] N. Nadarajah, R. Tharmarasa, M. McDonald, and T. Kirubarajan, "IMM forward filtering and backward smoothing for maneuvering target tracking," IEEE Transactions on Aerospace and Electronic Systems, vol. 48, no. 3, pp. 2673-2678, 2012.

[12] H. Qu, L. Pang, and S. Li, "A novel interacting multiple model algorithm," Signal Processing, vol. 89, no. 11, pp. 2171-2177, 2009.

[13] N. Seshadri and C.-E. W. Sundberg, "List Viterbi decoding algorithms with applications," IEEE Transactions on Communications, vol. 42, no. 2, pp. 313-323, 1994.

[14] T.-J. Ho, "A switched IMM-extended Viterbi estimator-based algorithm for maneuvering target tracking," Automatica, vol. 47, no. 1, pp. 92-98, 2011.

[15] X. R. Li and V. P. Jilkov, "Survey of maneuvering target tracking. Part I: dynamic models," IEEE Transactions on Aerospace and Electronic Systems, vol. 39, no. 4, pp. 1333-1364, 2003. 


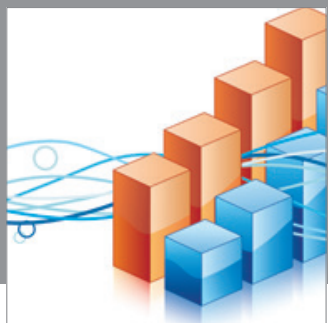

Advances in

Operations Research

mansans

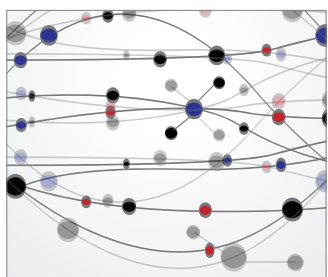

The Scientific World Journal
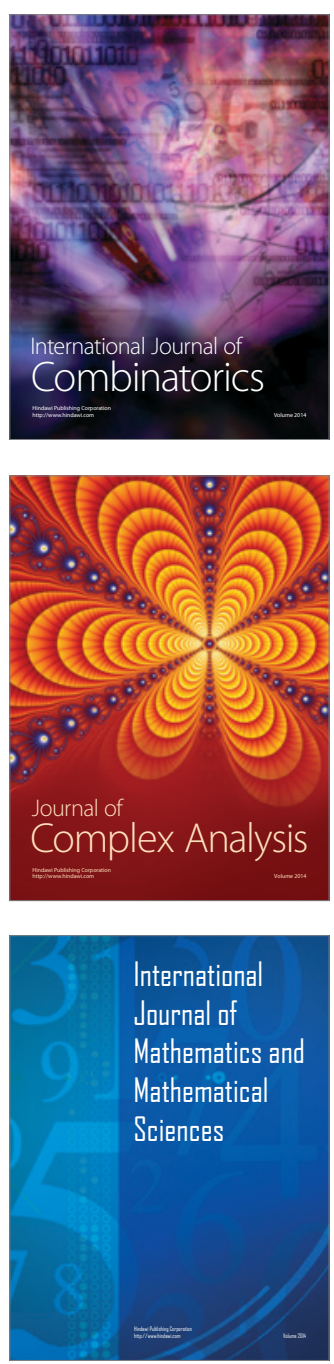
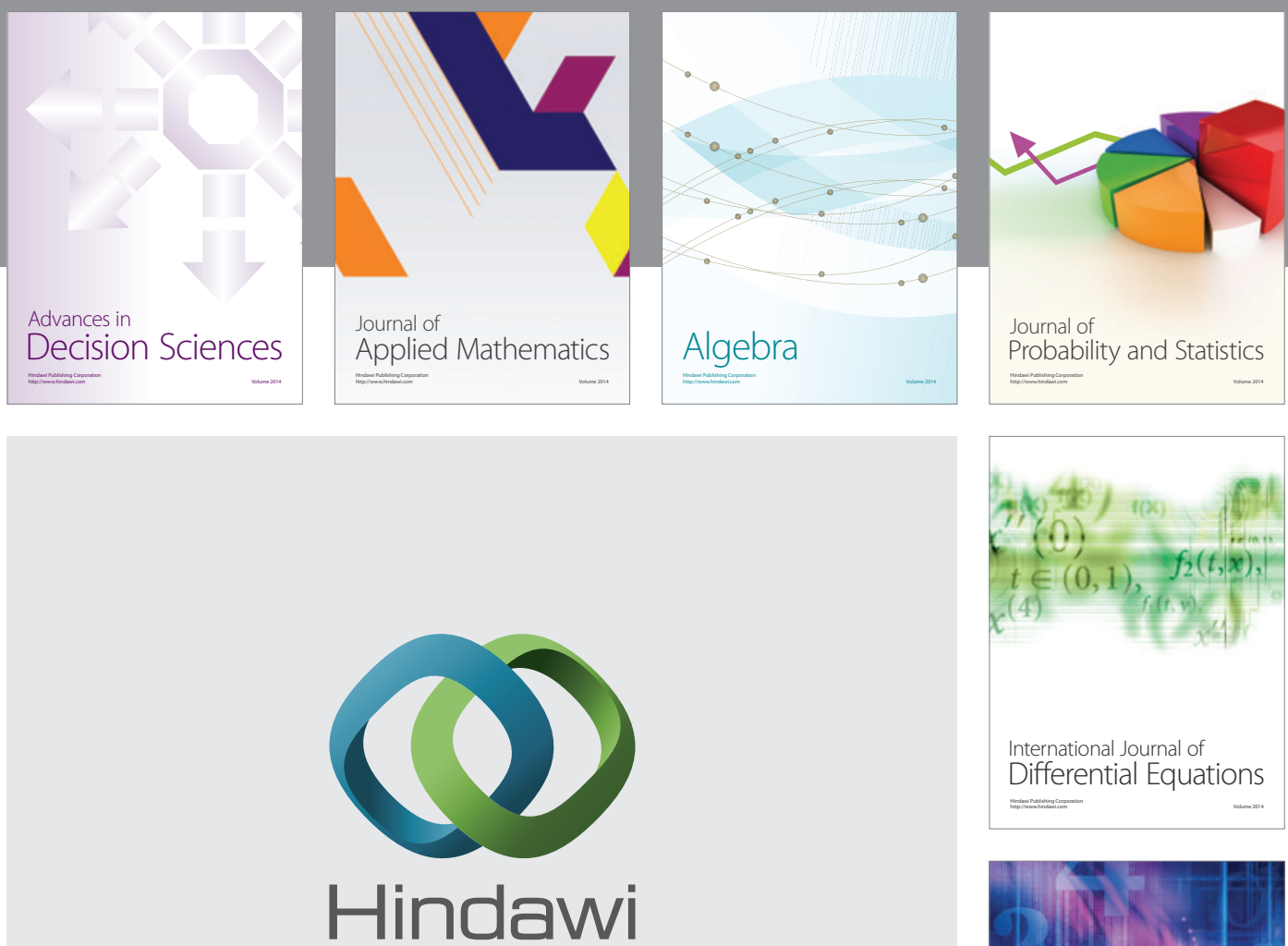

Submit your manuscripts at http://www.hindawi.com
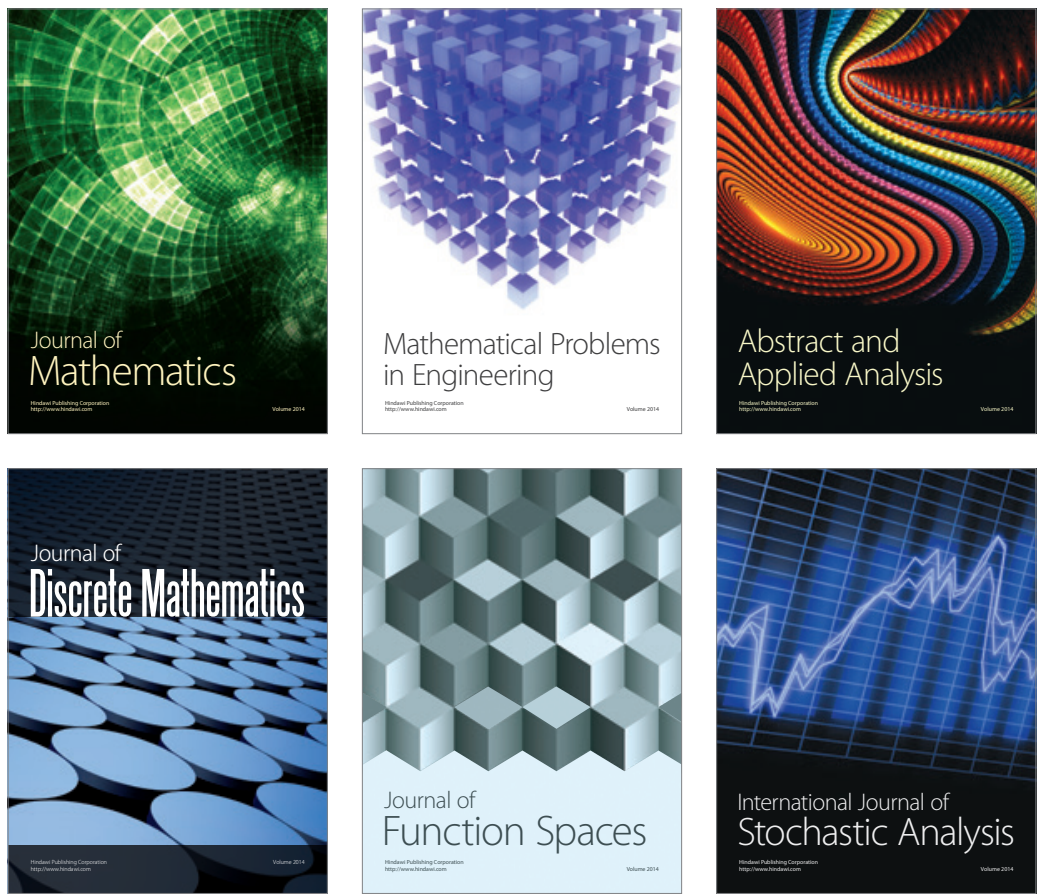

Journal of

Function Spaces

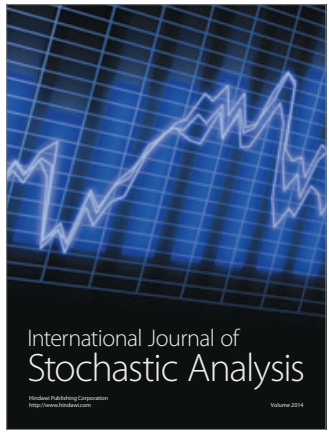

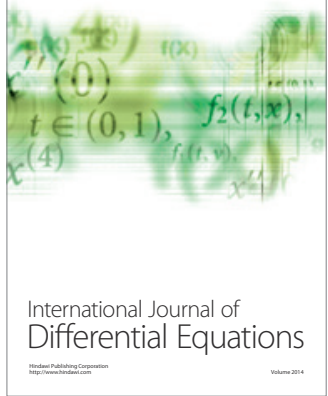
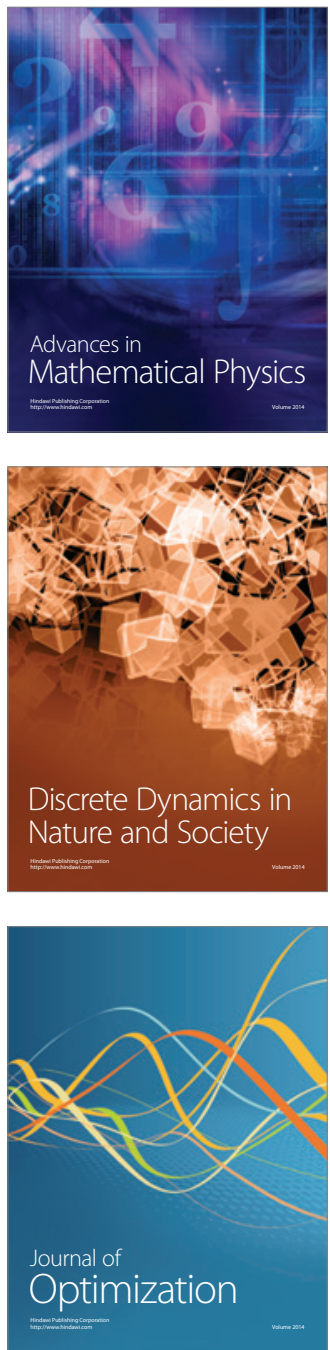